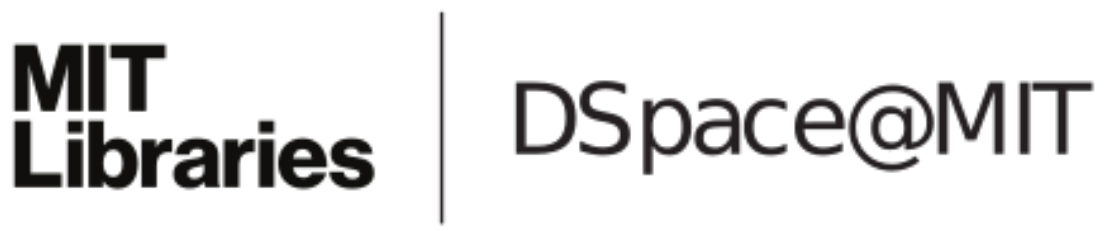

\author{
MIT Open Access Articles
}

\section{AN INITIAL MASS FUNCTION STUDY OF THE DWARF STARBURST GALAXY NGC 4214}

The MIT Faculty has made this article openly available. Please share how this access benefits you. Your story matters.

Citation: Andrews, J. E., D. Calzetti, R. Chandar, J. C. Lee, B. G. Elmegreen, R. C. Kennicutt, B. Whitmore, et al. "AN INITIAL MASS FUNCTION STUDY OF THE DWARF STARBURST GALAXY NGC 4214." The Astrophysical Journal 767, no. 1 (March 25, 2013): 51. (c) 2013 American Astronomical Society.

As Published: http://dx.doi.org/10.1088/0004-637x/767/1/51

Publisher: Institute of Physics/American Astronomical Society

Persistent URL: http://hdl.handle.net/1721.1/95421

Version: Final published version: final published article, as it appeared in a journal, conference proceedings, or other formally published context

Terms of Use: Article is made available in accordance with the publisher's policy and may be subject to US copyright law. Please refer to the publisher's site for terms of use. 


\title{
AN INITIAL MASS FUNCTION STUDY OF THE DWARF STARBURST GALAXY NGC 4214*
}

\author{
J. E. Andrews ${ }^{1}$, D. Calzetti ${ }^{1}$, R. Chandar ${ }^{2}$, J. C. LeE $^{3,4,14}$, B. G. Elmegreen ${ }^{5}$, R. C. Kennicutt ${ }^{6}$, \\ B. Whitmore ${ }^{3}$, J. S. Kissel ${ }^{7}$, Robert L. DA Silva ${ }^{8,15}$, Mark R. Krumholz ${ }^{8}$, R. W. O'Connell ${ }^{9}$, \\ M. A. Dopita ${ }^{10,11}$, JAY A. Frogel ${ }^{12}$, AND HwiHYUn KIM ${ }^{13}$ \\ ${ }^{1}$ Department of Astronomy, University of Massachusetts, Amherst, MA 01003, USA; \\ jandrews@astro.umass.edu, callzetti@astro.umass.edu \\ 2 Department of Physics and Astronomy, University of Toledo, Toledo, OH 43606, USA \\ ${ }^{3}$ Space Telescope Science Institute, 3700 San Martin Drive, Baltimore, MD 21218, USA \\ ${ }^{4}$ Spitzer Science Center, Caltech, Pasadena, CA 91125, USA \\ 5 IBM T. J. Watson Research Center, Yorktown Heights, NY, USA \\ ${ }^{6}$ Institute of Astronomy, Cambridge University, Cambridge, UK \\ ${ }^{7}$ Kavli Institute for Astrophysics and Space Research, Massachusetts Institute of Technology, Cambridge, MA 02139, USA \\ ${ }^{8}$ Department of Astronomy and Astrophysics, University of California, 1156 High Street, Santa Cruz, CA 95064, USA \\ ${ }^{9}$ Department of Astronomy, University of Virginia, P.O. Box 3818, Charlottesville, VA 22903, USA \\ ${ }^{10}$ Research School of Astronomy and Astrophysics, Australian National University, Cotter Road, Weston, ACT 2611, Australia \\ ${ }^{11}$ Astronomy Department, King Abdulaziz University, P.O. Box 80203, Jeddah, Saudi Arabia \\ 12 Galaxies Unlimited, 1 Tremblant Court, Lutherville, MD, USA \\ ${ }^{13}$ School of Earth and Space Exploration, Arizona State University, Tempe, AZ 85287-1404, USA \\ Received 2012 November 13; accepted 2013 February 18; published 2013 March 25
}

\begin{abstract}
The production rate of ionizing photons in young ( $\leqslant 8 \mathrm{Myr})$, unresolved stellar clusters in the nearby irregular galaxy NGC 4214 is probed using multi-wavelength Hubble Space Telescope WFC3 data. We normalize the ionizing photon rate by the cluster mass to investigate the upper end of the stellar initial mass function (IMF). We have found that within the uncertainties the upper end of the stellar IMF appears to be universal in this galaxy, and that deviations from a universal IMF can be attributed to stochastic sampling of stars in clusters with masses $\lesssim 10^{3} M_{\odot}$. Furthermore, we have found that there does not seem to be a dependence of the maximum stellar mass on the cluster mass. We have also found that for massive clusters, feedback may cause an underrepresentation in $\mathrm{H} \alpha$ luminosities, which needs to be taken into account when conducting this type of analysis.
\end{abstract}

Key words: galaxies: individual (NGC 4214) - galaxies: star clusters: general - galaxies: star formation stars: luminosity function, mass function - stars: massive

Online-only material: color figures

\section{INTRODUCTION}

The stellar initial mass function (IMF), the distribution of stellar masses in newly formed stellar populations, is essential for understanding the evolution and star formation histories of galaxies. Whether it is universal or dependent on environment has been a highly contested issue over the past few years. While IMF measurements in high-density environments such as the Milky Way and Magellanic Clouds have indicated an invariant IMF (Bastian et al. 2010; Massey 2003; Oey 2011), other claims of a non-universal IMF have been made (van Dokkum \& Conroy 2011; Wilkins et al. 2008; Fardal et al. 2007; Kroupa et al. 2011; Cappellari et al. 2012). In particular, star-forming dwarf galaxies may show a deficiency in the ionizing photon rate per unit UV or optical luminosity (Hoversten \& Glazebrook 2008; Lee et al. 2009; Meurer et al. 2009; Boselli et al. 2009; Gunawardhana et al. 2011). However, Fumagalli et al. (2011) and Weisz et al. (2012) have shown that stochasticity in populating the IMF or bursts of star formation can explain the observed variations in $L_{\mathrm{H} \alpha} / L_{\mathrm{FUV}}$ so that an unusual IMF is not required. Clearly this

\footnotetext{
* Based on observations made with the NASA/ESA Hubble Space Telescope, obtained at the Space Telescope Science Institute, which is operated by the Association of Universities for Research in Astronomy, Inc., under NASA contract NAS 5-26555. These observations are associated with program GO-11360.

14 Visiting Astronomer.

15 NSF Graduate Research Fellow.
}

is an unresolved issue that is essential to understanding the fundamental evolution of galaxies.

Traditional methods for IMF measurements in the Milky Way, Large Magellanic Cloud (LMC), and Small Magellanic Cloud (SMC) are to count individual stars in clusters $\leqslant 3-5$ Myr old that still retain their most massive stars (Anderson et al. 2009; Sabbi et al. 2008; Sirianni et al. 2000, for example). Generally only $20 \%$ of stellar clusters will survive early mass loss to live longer than 10 Myr (Lada \& Lada 2003, "infant mortality"), so catching them very early is essential for observing the full stellar population. Even nearby, significant crowding can cause these star counts to be incomplete, with high-mass stars and low-mass stars suffering from different selection biases: lowmass stars are generally harder to count, due to the inability to easily detect smaller, fainter stars and due to dynamical ejection of the low-mass stars, while massive stars may suffer from confusion due to their sinking toward the center of the cluster (Ascenso et al. 2009; Maíz Apellániz 2008). This method becomes progressively less effective at distances outside of the Magellanic Clouds ( $\sim 50 \mathrm{kpc})$, even with the resolution of the Hubble Space Telescope (HST).

As demonstrated in Calzetti et al. (2010), it is possible to constrain the upper end of the IMF in external galaxies without the use of individual star counts. Instead, a nearly coeval population that still contains the most massive members capable of producing ionizing photons can be constructed from the sum of unresolved young clusters in the galaxy. 


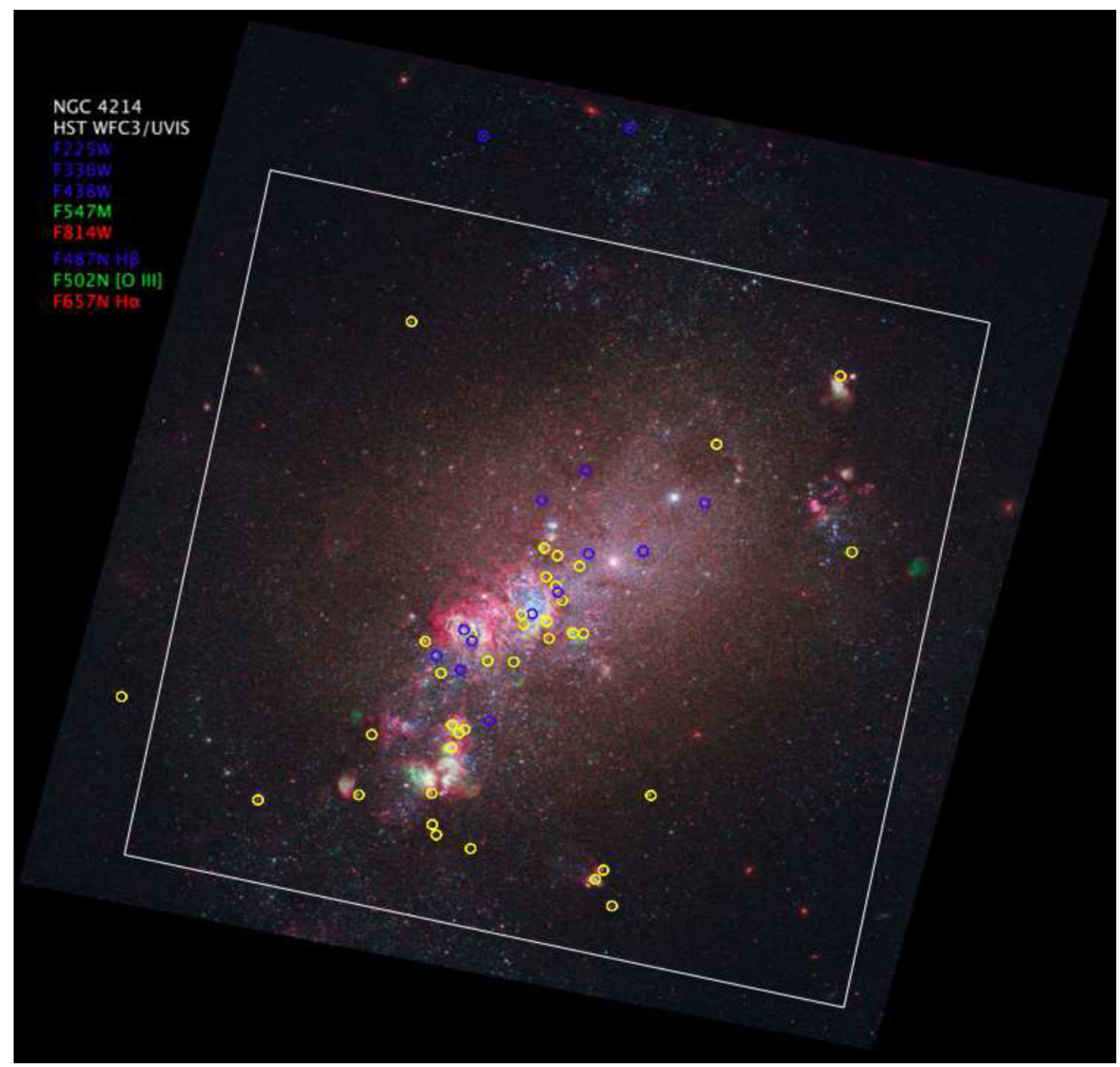

Figure 1. Color composite WFC3 image of NGC 4214 courtesy of Zoltan Levay (STScI-2011-14), R. O'Connell (GO 11360), and the WFC3 SOC. The footprint of the WFC3/IR images is shown in white. Blue circles are clusters with undetected $\mathrm{H} \alpha$ emission, and yellow circles are clusters with $\mathrm{H} \alpha$ emission. There are a total of 52 compact clusters.

(A color version of this figure is available in the online journal.)

Therefore, measuring $Q\left(\mathrm{H}^{0}\right)$, the hydrogen ionizing photon rate, in these young clusters is equivalent to measuring the number of massive stars. This method relies on normalizing the ionizing photon rate to the age-independent cluster mass, which is an extension of the method described in Corbelli et al. (2009). The treatment presented here can eliminate the need for agedependent bolometric luminosities, but does require that the cluster ages are accurately determined. The pilot study done on M51a by Calzetti et al. (2010) found that there was no obvious dependence of the upper mass end of the IMF on the mass of the star cluster down to $\sim 10^{3} M_{\odot}$, but a more extensive analysis including additional populations than M51a is needed for conclusive evidence. Specifically galaxies with star formation rates (SFRs) below the threshold for which IMF variances have been suggested $\left(\leqslant 0.1 M_{\odot} \mathrm{yr}^{-1}\right)$ need to be investigated. This paper aims to extend this study using a nearby galaxy with a lower SFR more similar to those dwarf galaxies which may be exhibiting a deficiency of ionizing photons.

NGC 4214 is an irregular, LMC-type, star-bursting galaxy located $\sim 3 \mathrm{Mpc}$ away (Dopita et al. 2010) with an $\mathrm{H} \alpha$ and UV SFR of $0.16 M_{\odot} \mathrm{yr}^{-1}$ and $0.22 M_{\odot} \mathrm{yr}^{-1}$, respectively (Lee et al. 2009, 2011; Kennicutt et al. 2008) as well as a sub-solar ( $Z \sim 0.25 Z_{\odot}$ ) metallicity (Kobulnicky \& Skillman 1996). The star formation history of the central region of NGC 4214 shows a strong increase in SFR starting $100 \mathrm{Myr}$ ago with a prominent peak at recent times $(\leqslant 10 \mathrm{Myr})$; despite this, less than $1 \%$ of the mass of the galaxy is due to the current star formation event (Williams et al. 2011). Due to its proximity and recent star-forming activity, NGC 4214 is an ideal test bed for the upper end of the IMF (uIMF). In Section 2, we will discuss the observations and cluster selection criteria, in Section 3 we will present the models and age and mass determinations, and in Section 4 we discuss the results.

\section{OBSERVATIONS AND CLUSTER SELECTION}

Observations were taken with HST WFC3/UVIS and WFC3/IR as part of GO 11360 (PI: O'Connell). The observations on which we concentrate here include $F 225 W$ (1665 s), $F 336 W(1683$ s), F438W (1530 s), F547M (1820 s), F657N (1592 s), F814W (1339 s), F110W (1198 s), and $F 128 N$ (1198 s), as shown in Figure 1. For ease of discussion, we 
will refer to these as NUV, $U, B, V, \mathrm{H} \alpha, I, J$, and $\mathrm{P} \beta$, respectively. Each flat-fielded image was co-added, cosmic rays were removed, and corrections for distortion were made using the task MULTIDRIZZLE into a final pixel scale of 0 '.0396 pixel $^{-1}$. This corresponds to $0.58 \mathrm{pc} \mathrm{pixel}^{-1}$ at a distance of $3 \mathrm{Mpc}$. See Dopita et al. (2010) for a full explanation of the reduction procedure, including the creation of continuum-subtracted $\mathrm{H} \alpha$ images. A similar procedure was adopted to create a continuum-subtracted $\mathrm{P} \beta$ image, which covers $\sim 75 \%$ of the UVIS field of view. The footprint of the IR field of view is also shown as a white outline in Figure 1.

Cluster candidates were identified using a technique similar to that described in Chandar et al. $(2010,2011)$ for M51 and M83. Aperture photometry was performed on the wide and medium band images using the IRAF task PHOT with an aperture of 3 pixels, and a background annulus between 10 and 13 pixels. The aperture corrections were addressed similarly as was done on clusters in M83 from Chandar et al. (2010). In their study they chose two different methods for aperture correction, both of which rely on the concentration index $(C$, the difference in magnitudes between 3 pixel and 0.5 pixel radius). Method one used a single value for the aperture corrections of point sources $(C<2.3)$ and a slightly larger value for extended sources $(C>2.3)$. For this sample we chose to use their second approach, which is to use an aperture correction equation for extended objects with $2.3<C \leqslant 3.4$. Photometric conversion from counts $\mathrm{s}^{-1}$ to erg $\mathrm{cm}^{-2} \mathrm{~s}^{-1}$ was accomplished using the filterdependent PHOTFLAM values provided by the STScI Web site. Galactic foreground extinction of $E(B-V)=0.02$ was corrected using the Milky Way extinction curve from Fitzpatrick (1999).

Due to the more extended nature of $\mathrm{H}$ II regions surrounding the stellar clusters, aperture sizes that scaled with the cluster mass according to the expected Strömgren radius were used to measure the hydrogen recombination lines on the continuumsubtracted $\mathrm{H} \alpha+[\mathrm{N}$ II $]$ and $\mathrm{P} \beta$ images. As was done in Calzetti et al. (2010), a radius of about $0.35 R_{\text {Strömgren }}$ was selected due to the crowding of the clusters, which corresponds to a range between 6 and 10 pixels. This radius is sufficiently larger than the PSFs for both the $\mathrm{H} \alpha$ and $\mathrm{P} \beta$ images, so there are no concerns of PSF variations. The local background was subtracted using a 3 pixel wide annulus centered on the cluster outside of the aperture radius in order to avoid contamination from other diffuse emission. Aperture corrections were calculated from a few, very isolated sources and were applied to the other regions. We found that an additional correction of 1.30 times the flux was needed to go from a 20 pixel aperture to an "infinite" aperture. Contamination from $[\mathrm{N}$ II] was removed using the average galactic $[\mathrm{N}$ II $] / \mathrm{H} \alpha$ ratio of 0.11 from Kobulnicky \& Skillman (1996). The corrections for the ionized gas extinctions were measured region by region using the WFC3/IR $\mathrm{P} \beta$ image for those regions where the IR and UVIS overlap (see Figure 1) using the formulation in Calzetti et al. (2000) and were applied to the $\mathrm{H} \alpha$ luminosities.

The cluster catalog was populated by the combination of two methods. The first, and most robust, was using the automated method discussed in depth in Chandar et al. (2010) which accounts for roughly $80 \%$ of the cluster catalog. To make sure all clusters are accounted for, we also use a manual selection procedure from a careful examination of the WFC3 images. This ensures we identify clusters in crowded regions or clusters near a bright star which may have been missed in the automated process. In total we have identified $\sim 400$ cluster candidates.

\section{ANALYSIS}

In this section, we will present an in-depth discussion of the analysis procedure used in this paper, and a detailed description of the filter convolution is included in the Appendix. For the mass and age determination of the clusters we have used broadband photometry (without the $\mathrm{H} \alpha$ included) to determine ages and masses of the clusters using both a canonical and truncated IMF from stochastic and deterministic stellar models. The best-fit model-derived masses of clusters with ages less than $8 \mathrm{Myr}$ are then binned into three distinct mass bins. Within these mass bins the masses are summed and the $\mathrm{H} \alpha$ luminosities are summed to determine the $L_{\mathrm{H} \alpha} / M_{\mathrm{cl}}$ ratios for various cluster masses $\left(\left\langle L_{\mathrm{H} \alpha} / M_{\mathrm{cl}}\right\rangle=\sum_{i} L_{\mathrm{H} \alpha i} / \sum_{i} M_{\mathrm{cli}}\right)$. These ratios are then compared to predicted models with two different assumptions about the IMF, the canonical one and the variable upper mass limit (Kroupa \& Weidner 2003, see Section 4), in order to constrain the IMF of NGC 4214.

\subsection{The Models}

In order to accurately determine ages and masses of the clusters, the spectral energy distributions (SEDs) created from the photometry of the NUV, $U, B, V, I$ observations were compared to stellar synthesis models. While the norm has been to use deterministic models such as STARBURST99 (SB99; Leitherer et al. 1999), these models assume a fully sampled stellar IMF, which for smaller mass clusters could lead to the inclusion of unphysical fractions of stars. With stellar clusters of high masses $\left(\geqslant 1 \times 10^{4} M_{\odot}\right)$, we expect the IMF to be fully populated (Elmegreen 2006; da Silva et al. 2012), so this is not a problem, but, as the cluster masses decrease, massive stars are less likely to be formed and massive stellar populations are not fully represented. In order to properly measure parameters for low-mass clusters $\left(\sim 500-5000 M_{\odot}\right)$, it is then important to turn to stochastic modeling. For this paper, we will mainly focus on the stochastic models of SLUG (da Silva et al. 2012, Stochastically Lighting Up Galaxies) which performs the synthesis of composite populations using individual stellar clusters which are stochastically populated with stars, using the IMF as a probability distribution function. Other stochastic models are presented in Popescu \& Hanson (2010), but are not used in this study. According to Cerviño \& Luridiana (2004, 2006), clusters with masses below $10^{3} M_{\odot}$ and ages less than $10^{7}$ yr may be susceptible to color biases from deterministic stellar synthesis models. NGC 4214 has numerous small clusters making it pertinent that stochastic models be used. As a check and comparison, we also employ the deterministic SB99 models using the same input parameters and use outputs which contain both stellar and nebular emission as cautioned by Reines et al. (2010).

Both sets of models use a Kroupa IMF between 0.08 and $120 M_{\odot}$ (Kroupa 2001), Padova asymptotic giant branch tracks with $z=0.004$, and assume that the clusters form in a single instantaneous burst. For the truncated SLUG models, where the maximum mass was only allowed to be $30 M_{\odot}$, a Salpeter IMF was used. The SB99 models contain ages between 1 and $200 \mathrm{Myr}$ in timesteps of every $1 \mathrm{Myr}$ for models between 1 and $20 \mathrm{Myr}$, and every $25 \mathrm{Myr}$ for models between 25 and $200 \mathrm{Myr}$ (to easily distinguish old clusters.) New SB99 models were generated between 2 and $8 \mathrm{Myr}$ with time steps of $0.2 \mathrm{Myr}$ for more accurate comparison with SLUG models. The SLUG models include about 40,000 cluster templates with ages from $0.01 \mathrm{Myr}$ to $20 \mathrm{Myr}$ with a cluster mass of $1 \times 10^{3} M_{\odot}$ (Figure 2 ). 


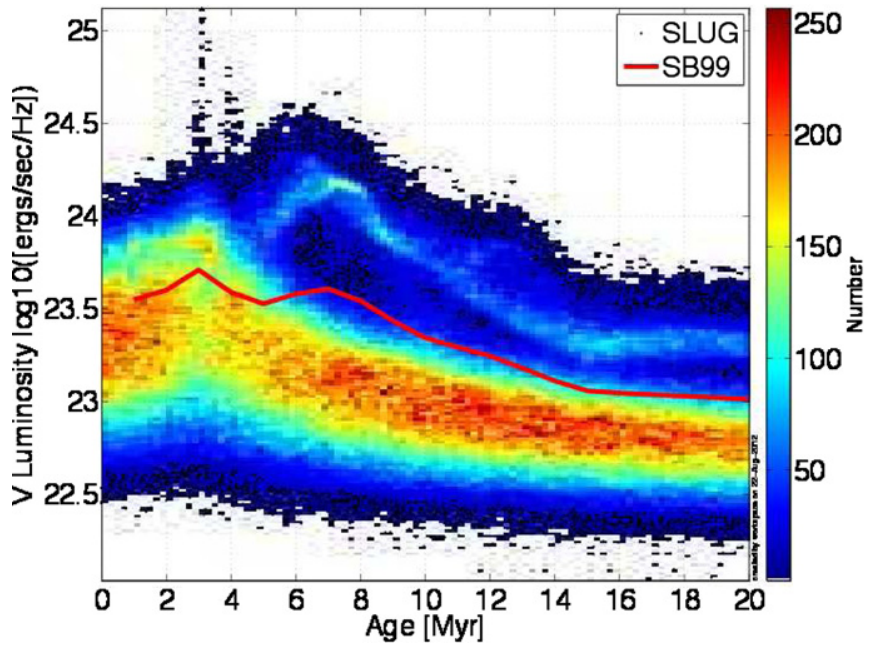

Figure 2. Comparison of $V$-band luminosities of $1 \times 10^{3} M_{\odot}$ SLUG models from 1 to $20 \mathrm{Myr}$ with SB99 (red line) with $A_{V}=0$. The magnitude of scatter from the stochastic models will also create a scatter of cluster mass. Note that the arithmetic mean of the SLUG models matches the SB99 models, but that the geometric mean is slightly lower.

(A color version of this figure is available in the online journal.)

Tests have been run on a subset of clusters using $5 \times 10^{2}, 1 \times$ $10^{3}$, and $3 \times 10^{3} M_{\odot}$ SLUG models and we have found that the difference in inferred ages and masses of the same cluster among the various models is small, and is already encompassed by the uncertainties generated within one $1 \times 10^{3} M_{\odot}$ model, which is described below. Therefore, to simplify the analysis and most accurately reflect the masses of the clusters in NGC 4214, we use only $1 \times 10^{3} M_{\odot}$ SLUG models for our cluster sample. SLUG models do not allow for binarity, but a recent study by Eldridge (2012) indicates that at masses $\geqslant 10^{3} M_{\odot}$, the scatter in $L_{\mathrm{H} \alpha} / M_{\mathrm{cl}}$ is the same between models that use single stars and those that introduce binaries, so this should not introduce additional uncertainties.

\subsection{Age and Mass Determination}

To estimate the age, mass, and extinction of each cluster we employ a reduced $\chi^{2}$ fitting technique between both the SLUG and SB99 models and the cluster photometry. To do this, we have used the Yafit (Yet Another Fitting Tool ${ }^{16}$ ) curve fitting tool, which was created to fit photometry with model SEDs. It provides both a graphical and a numerical output containing the reduced $\chi^{2}$ value and the scaling factor between model and observation. The ensemble of photometry for each cluster was compared to both SLUG and SB99 models spanning the complete reddening range between $0 \leqslant E(B-V) \leqslant 0.40$. Note, we do not use the $\mathrm{H} \alpha$ filter as part of the fit, as we do not want to bias our sample based on the presence or absence of $\mathrm{H} \alpha$ emission.

After all of the observations were compared against each model, the fits for each cluster were then organized into increasing reduced $\chi^{2}$ values. It is important here to point out that there is no single solution for the age and extinction of the cluster, but instead there is a range of best fits which could produce the model fit. In Figure 3, we show both the SED fits and the range of ages and extinctions spanned by the model fits to the youngest ( $\sim 2 \mathrm{Myr})$ and oldest ( $\sim 7.5 \mathrm{Myr})$ clusters in our sample. By using the large range of ages

\footnotetext{
16 http://www.star.bris.ac.uk/mbt/yafit/
}

and extinction consistent with the model fits, the actual mass distribution has been spread over a large range of values and only produces a peak at the most probable value. Therefore, we allowed the $\chi^{2}$ values to range between 0 and 1, as was done in Pasquali et al. (2003), and include all ages, extinctions, and therefore corresponding masses within that range. Masses were determined using the scaling factor output by Yafit and attributing error bars consistent with the error bars in the ages and extinction values. As with Calzetti et al. (2010), we also find that changing the upper mass limit of the IMF in both the SLUG and SB99 models from 120 to $30 M_{\odot}$ increases the mass estimates by roughly 2.5 . To double check our initial results, comparisons with previous studies which have determined ages and masses of some of the clusters in NGC 4214 were conducted and found to be comparable. For example, the young massive cluster located at the center of NGC 4214 (R.A. $=12^{\mathrm{h}} 15^{\mathrm{m}} 39^{\mathrm{s}} .44$, decl. $=36^{\circ} 19^{\prime} 34^{\prime \prime} .94$ ), noted here as Cluster 1, has age and mass estimates of 4-5 Myr and $2.7 \pm 0.4 \times 10^{4} M_{\odot}$ (MacKenty et al. 2000; Leitherer et al. 1996) using a Salpeter IMF between 1 and $100 M_{\odot}$. Our best estimate, from the best fit from both SLUG and SB99, is 4.2 and $4.8 \mathrm{Myr}$, respectively, with a corresponding best-fit mass of $9.3 \pm 4$ and $9.7 \pm 3 \times 10^{4} M_{\odot}$, which is consistent with these previous studies within $2 \sigma$. Unfortunately the cluster has blown an extensive asymmetric bubble, clearing the surrounding region of much of its hydrogen gas and allowing more than half of the ionizing photons to escape (MacKenty et al. 2000), making accurate measurements of $L_{\mathrm{H} \alpha}$ extremely difficult. For completeness purposes, we will use the $\mathrm{H} \alpha$ flux for Cluster 1 (I-As) quoted in MacKenty et al. (2000), which gives an $\mathrm{H} \alpha$ luminosity of $8.4 \times 10^{37} \mathrm{erg} \mathrm{s}^{-1}$.

By $\leqslant 8$ Myr, the compact $\mathrm{H}$ II region surrounding stellar clusters has expanded into a shell structure which disperses into the ISM (Whitmore et al. 2011) and massive stars capable of producing ionizing photons $\left(>15-20 M_{\odot}\right)$ have disappeared. For example, SB99 models using the parameters listed above show that the $\mathrm{H} \alpha$ luminosity at $8 \mathrm{Myr}$ is only $2.5 \%$ of $L_{\mathrm{H} \alpha}$ at 2 Myr. Therefore, it is necessary to exclude all clusters $>8 \mathrm{Myr}$ in order to keep objects in which the H II region is still density bound and the massive stars are still retained. This ensures that uncertainties in the ionizing photon rate are reduced and that constraints can still be made on the upper end of the IMF. As will be discussed below and is shown in Figure 4, including ages greater than $6 \mathrm{Myr}$ may already be too old for this type of analysis. We must also be careful of confusion, for example, including multiple objects which may share the same $\mathrm{H}$ iI region. For this reason, we have excluded those clusters which may share in ionizing common gas, removing clusters which are in excessively crowded regions which would hinder the correct measurement of $L_{\mathrm{H} \alpha}$. This has left us with a total of 89 clusters between the ages of 2 and $8 \mathrm{Myr}, 52$ of which have masses that are $\geqslant 500 M_{\odot}$, and either have a measured $\mathrm{H} \alpha$ luminosity (38) or have an $\mathrm{H} \alpha$ luminosity that is non-detectable down to the $3 \sigma$ limit of $1.6 \times 10^{35} \mathrm{erg} \mathrm{s}^{-1}$. Ten of these objects (Table 1, footnote c) have PSFs that are consistent with a single massive star, yet their SEDs require the presence of multiple stars to be fully accounted for in flux. These low-multiplicity clusters tend to be among our lowest mass systems (Table 1) and may be the NGC 4214 equivalent of the Trapezium cluster in Orion, which has a $1-1.5 \mathrm{pc}$ size and a handful of $15-30 M_{\odot}$ stars. Therefore, they have still been included in the sample and are indicated in Table 1 with the rest of the clusters. Table 1 also includes the corresponding ages, extinctions, and masses 

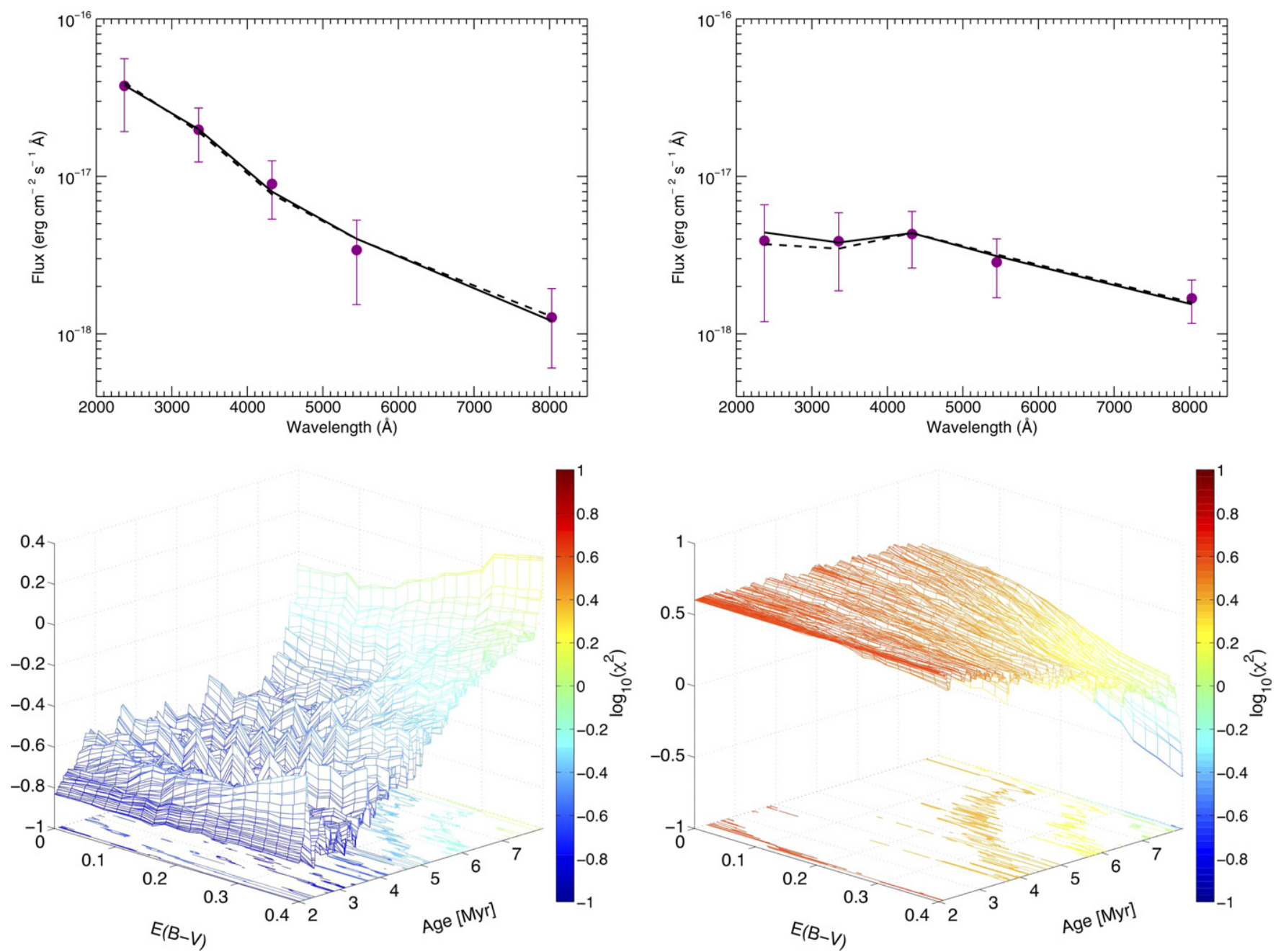

Figure 3. Top: SED model fits for a $\sim 2$ Myr cluster (left, cluster 402 in Table 1) and a $\sim 7.5$ Myr cluster (right, cluster 188 in Table 1). Each fit uses an $E(B-V)$ of 0.06 and 0.40 , respectively. SLUG models are indicated by a solid line, and SB99 models by a dashed line. The large uncertainties in the photometry can allow for a large range of fits with an acceptable $\chi^{2}$ value. Bottom: contours for the reduced $\chi^{2}$ values for various age and attenuation values for a $\sim 2$ Myr cluster (left) and a $\sim 7.5$ Myr cluster (right).

(A color version of this figure is available in the online journal.)
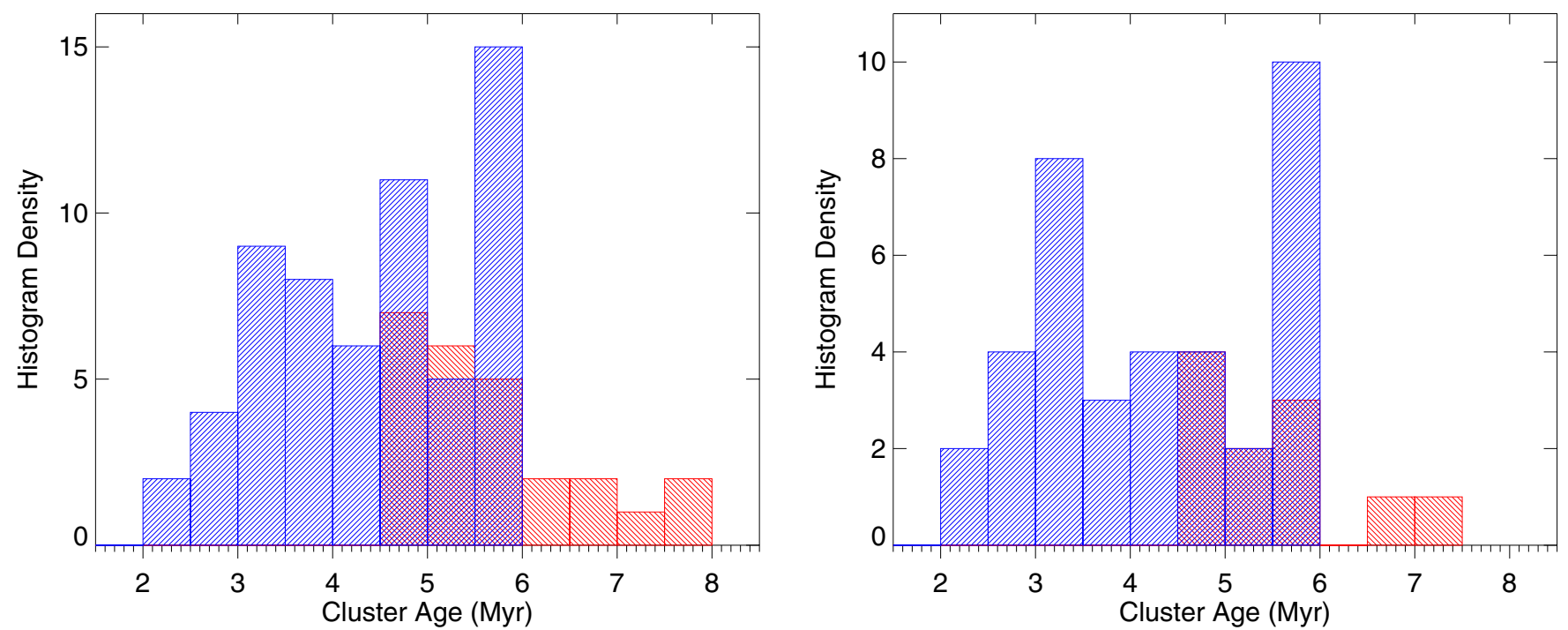

Figure 4. Histograms of relative frequencies of best-fit cluster ages for all young clusters (left) and clusters with masses $>500 M_{\odot}$ (right). The blue histogram indicates those clusters with measured $\mathrm{H} \alpha$ emission, while the red indicates those with only upper limits. From both plots it is apparent that clusters $<4.5 \mathrm{Myr}$ will still retain $\mathrm{H} \alpha$ emission, while those $>6 \mathrm{Myr}$ will not. Cluster with ages between 4.5 and $6 \mathrm{Myr}$ have a lower probability of producing $\mathrm{H} \alpha$ emission. These plots exclude those clusters with masses $>10^{4} M_{\odot}$ as they may not be fully explored by the SLUG models and are far more likely to have expelled their surrounding hydrogen gas at a much younger age.

(A color version of this figure is available in the online journal.) 
Table 1

Ages, Masses, and Extinctions of Clusters

\begin{tabular}{|c|c|c|c|c|c|c|c|c|c|c|}
\hline ID & $\begin{array}{l}\alpha(\mathrm{J} 2000) \\
12^{\mathrm{h}} 15^{\mathrm{m}}+\end{array}$ & $\begin{array}{c}\delta(\mathrm{J} 2000) \\
36^{\circ}+\end{array}$ & $\begin{array}{l}\text { Age } \\
(\mathrm{Myr})\end{array}$ & $\begin{array}{c}\text { SLUG } \\
\text { Mass } \\
\left(10^{3} M_{\odot}\right)\end{array}$ & $E(B-V)$ & $\begin{array}{l}\text { Age } \\
(\mathrm{Myr})\end{array}$ & $\begin{array}{c}\text { SB99 } \\
\text { Mass } \\
\left(10^{3} M_{\odot}\right)\end{array}$ & $E(B-V)$ & $\begin{array}{c}L_{\mathrm{H} \alpha}{ }^{\mathrm{a}} \\
\left(\mathrm{erg} \mathrm{s}^{-1}\right)\end{array}$ & $\begin{array}{c}\text { Other } \\
\text { Names }^{\mathrm{b}}\end{array}$ \\
\hline$\overline{55^{\mathrm{c}}}$ & 39.278 & 1955.94 & $5.9 \pm 0.9$ & $0.50_{0.6}^{0.2}$ & 0.24 & 4.0 & 0.21 & 0.16 & $1.6 \mathrm{E}+35$ & \\
\hline $145^{\mathrm{c}}$ & 40.065 & 1914.13 & $4.6 \pm 3.0$ & $0.54_{1.2}^{0.5}$ & 0.16 & 5.0 & 0.12 & 0.2 & $1.6 \mathrm{E}+35$ & \\
\hline 115 & 37.723 & 1946.42 & $6.6 \pm 2.0$ & $0.57_{2.5}^{0.4}$ & 0.32 & 4.0 & 0.50 & 0.4 & $1.6 \mathrm{E}+35$ & \\
\hline $68^{\mathrm{c}}$ & 37.008 & 1954.39 & $4.7 \pm 3.0$ & $0.60_{3.7}^{0.6}$ & 0.22 & 5.0 & 0.33 & 0.22 & $1.6 \mathrm{E}+35$ & \\
\hline 63 & 40.559 & 1924.44 & $5.6 \pm 2.3$ & $0.70_{2.0}^{0.7}$ & 0.22 & 5.4 & 0.20 & 0.12 & $1.6 \mathrm{E}+35$ & \\
\hline $159^{\mathrm{c}}$ & 39.072 & 1938.38 & $4.7 \pm 3.0$ & $0.86_{2.5}^{0.9}$ & 0.3 & 5.6 & 0.20 & 0.26 & $1.6 \mathrm{E}+35$ & \\
\hline 188 & 37.928 & 2105.26 & $7.5 \pm 0.8$ & $2.10_{2.1}^{2.0}$ & 0.4 & 7.6 & 0.40 & 0.34 & $1.6 \mathrm{E}+35$ & \\
\hline 27 & 38.508 & 1945.84 & $5.9 \pm 1.3$ & $2.22_{5.6}^{1.3}$ & 0.32 & 4.0 & 1.00 & 0.26 & $1.6 \mathrm{E}+35$ & \\
\hline 17 & 40.186 & 2103.80 & $5.2 \pm 1.7$ & $2.44_{3.4}^{2.4}$ & 0.06 & 5.8 & 0.49 & 0.02 & $1.6 \mathrm{E}+35$ & \\
\hline 34 & 38.653 & 2000.20 & $5.4 \pm 1.9$ & $2.73_{1.1}^{2.7}$ & 0.12 & 5.4 & 0.46 & 0.1 & $1.6 \mathrm{E}+35$ & \\
\hline 2 & 40.372 & 1929.87 & $3.1 \pm 0.5$ & $13.04_{2.7}^{0.1}$ & 0.06 & 4.8 & 3.60 & 0.04 & $1.6 \mathrm{E}+35$ & $\mathrm{I}-\mathrm{B} 2 \mathrm{n}$ \\
\hline 3 & 40.487 & 1931.71 & $2.6 \pm 0.5$ & $20.03_{0.9}^{2.7}$ & 0.08 & 4.8 & 4.58 & 0.08 & $1.6 \mathrm{E}+35$ & I-Bs \\
\hline 4 & 40.926 & 1927.09 & $2.9 \pm 1.5$ & $20.71_{1.9}^{5.0}$ & 0.06 & 5.2 & 3.77 & 0.06 & $1.6 \mathrm{E}+35$ & \\
\hline 140 & 40.925 & 1854.40 & $5.9 \pm 2.7$ & $0.49_{2.1}^{0.4}$ & 0.38 & 4.0 & 0.21 & 0.3 & $2.3 \mathrm{E}+36$ & \\
\hline 340 & 40.555 & 1912.84 & $3.7 \pm 1.7$ & $0.52_{2.0}^{2.5}$ & 0.16 & 3.8 & 0.38 & 0.16 & $3.1 \mathrm{E}+37$ & II-C1n \\
\hline $348^{\mathrm{c}}$ & 38.214 & 1840.35 & $3.1 \pm 1.7$ & $0.57_{0.4}^{0.5}$ & 0 & 4.6 & 0.17 & 0 & $3.7 \mathrm{E}+36$ & \\
\hline $359^{\mathrm{c}}$ & 39.226 & 1941.53 & $3.6 \pm 1.5$ & $0.57_{6.9}^{0.3}$ & 0.2 & 3.2 & 1.01 & 0.22 & $8.8 \mathrm{E}+36$ & \\
\hline 345 & 40.985 & 1855.60 & $2.7 \pm 2.4$ & $0.61_{3.5}^{0.6}$ & 0.28 & 4.2 & 0.39 & 0.28 & $4.0 \mathrm{E}+36$ & \\
\hline $74^{\mathrm{c}}$ & 38.679 & 1944.18 & $5.6 \pm 2.7$ & $0.65_{2.0}^{0.6}$ & 0.24 & 5.2 & 0.19 & 0.16 & $2.3 \mathrm{E}+36$ & \\
\hline 25 & 39.566 & 1932.93 & $3.5 \pm 1.2$ & $0.66_{0.7}^{0.5}$ & 0.14 & 3.2 & 0.39 & 0.14 & $5.1 \mathrm{E}+37$ & $\mathrm{I}-\mathrm{A} 5 \mathrm{n}$ \\
\hline $402^{\mathrm{c}}$ & 38.659 & 1931.17 & $2.2 \pm 1.5$ & $0.69_{0.9}^{0.6}$ & 0.06 & 3.6 & 0.22 & 0.08 & $5.1 \mathrm{E}+36$ & $\mathrm{I}-\mathrm{C} 1 \mathrm{n}$ \\
\hline 46 & 40.908 & 1923.26 & $2.9 \pm 1.9$ & $0.72_{0.5}^{0.7}$ & 0 & 5.2 & 0.13 & 0 & $7.0 \mathrm{E}+35$ & \\
\hline $125^{\mathrm{c}}$ & 45.614 & 1917.81 & $5.6 \pm 3.0$ & $0.76_{0.8}^{0.8}$ & 0.22 & 5.4 & 0.10 & 0.14 & $1.1 \mathrm{E}+37$ & \\
\hline 58 & 39.713 & 1925.88 & $4.3 \pm 2.7$ & $0.77_{1.1}^{0.7}$ & 0.04 & 4.8 & 0.13 & 0.04 & $9.7 \mathrm{E}+35$ & \\
\hline 24 & 39.613 & 1934.57 & $4.9 \pm 1.8$ & $0.78_{5.8}^{0.7}$ & 0.18 & 5.2 & 0.86 & 0.14 & $7.3 \mathrm{E}+36$ & \\
\hline 417 & 41.921 & 1912.33 & $5.9 \pm 3.0$ & $0.80_{7.3}^{0.7}$ & 0.22 & 5.0 & 0.62 & 0.14 & $3.9 \mathrm{E}+36$ & \\
\hline 328 & 34.729 & 2017.91 & $5.9 \pm 2.8$ & $0.85_{4.7}^{0.8}$ & 0.2 & 4.0 & 0.35 & 0.12 & $2.8 \mathrm{E}+37$ & \\
\hline 39 & 43.649 & 1900.15 & $2.9 \pm 1.6$ & $0.88_{0.4}^{0.8}$ & 0 & 5.4 & 0.17 & 0 & $3.6 \mathrm{E}+36$ & \\
\hline 384 & 39.067 & 1945.63 & $5.9 \pm 1.5$ & $0.93_{1.3}^{0.8}$ & 0.24 & 4.0 & 0.38 & 0.16 & $1.1 \mathrm{E}+37$ & \\
\hline 21 & 41.303 & 2029.18 & $4.7 \pm 1.5$ & $0.94_{2.6}^{0.8}$ & 0.04 & 5.0 & 0.34 & 0.02 & $9.5 \mathrm{E}+36$ & \\
\hline 360 & 38.996 & 1937.41 & $4.5 \pm 2.0$ & $0.95_{7.8}^{0.7}$ & 0.18 & 4.0 & 0.95 & 0.16 & $7.5 \mathrm{E}+37$ & I-A3n \\
\hline 326 & 42.110 & 1901.12 & $4.0 \pm 1.5$ & $1.14_{3.6}^{0.9}$ & 0.4 & 4.0 & 0.77 & 0.38 & $3.9 \mathrm{E}+37$ & IXn \\
\hline 83 & 41.017 & 1901.41 & $5.9 \pm 2.8$ & $1.18_{5.2}^{1.0}$ & 0.4 & 5.0 & 0.58 & 0.32 & $2.2 \mathrm{E}+37$ & II-A \\
\hline 358 & 39.285 & 1947.16 & $5.1 \pm 1.5$ & $1.26_{4.1}^{1.0}$ & 0.12 & 5.0 & 0.85 & 0.1 & $2.5 \mathrm{E}+37$ & I-D2n \\
\hline 352 & 38.482 & 1845.42 & $3.1 \pm 0.5$ & $1.31_{0.4}^{1.2}$ & 0 & 4.6 & 0.39 & 0 & $2.1 \mathrm{E}+37$ & \\
\hline 341 & 40.611 & 1912.53 & $3.5 \pm 1.5$ & $1.35_{4.3}^{1.2}$ & 0.14 & 3.2 & 0.78 & 0.14 & $3.9 \mathrm{E}+37$ & II-Cln \\
\hline 362 & 38.809 & 1931.27 & $3.4 \pm 1.2$ & $1.53_{1.7}^{0.5}$ & 0.12 & 4.4 & 3.39 & 0.08 & $1.6 \mathrm{E}+37$ & $\mathrm{I}-\mathrm{C} 1 \mathrm{n}$ \\
\hline 357 & 34.527 & 1946.39 & $5.6 \pm 2.0$ & $1.58_{5.0}^{0.4}$ & 0.08 & 5.0 & 0.57 & 0 & $2.5 \mathrm{E}+36$ & \\
\hline 338 & 40.700 & 1909.83 & $6.0 \pm 0.4$ & $1.62_{1.0}^{0.1}$ & 0 & 6.2 & 5.75 & 0.08 & $1.0 \mathrm{E}+38$ & II-B \\
\hline 81 & 39.087 & 1939.71 & $4.7 \pm 2.2$ & $1.63_{6.0}^{1.3}$ & 0.4 & 5.0 & 0.90 & 0.4 & $8.6 \mathrm{E}+35$ & \\
\hline $434^{\mathrm{c}}$ & 36.612 & 2006.30 & $3.4 \pm 1.7$ & $1.77_{1.6}^{1.3}$ & 0.38 & 4.8 & 0.43 & 0.1 & $2.2 \mathrm{E}+36$ & \\
\hline 15 & 40.119 & 1926.67 & $3.4 \pm 1.5$ & $2.16_{0.8}^{2.0}$ & 0.02 & 4.8 & 0.57 & 0.04 & $1.6 \mathrm{E}+36$ & \\
\hline 349 & 38.358 & 1847.05 & $2.5 \pm 1.5$ & $2.66_{0.4}^{2.2}$ & 0 & 4.8 & 0.56 & 0.22 & $1.2 \mathrm{E}+37$ & \\
\hline 100 & 40.398 & 1851.19 & $5.5 \pm 2.8$ & $2.67_{2.1}^{2.6}$ & 0.28 & 5.8 & 0.26 & 0.2 & $7.9 \mathrm{E}+35$ & \\
\hline 353 & 37.617 & 1900.90 & $3.3 \pm 2.2$ & $3.08_{5.5}^{3.0}$ & 0.28 & 4.6 & 1.20 & 0.26 & $1.9 \mathrm{E}+37$ & \\
\hline 11 & 40.384 & 1930.80 & $5.4 \pm 0.7$ & $3.39_{3.2}^{2.8}$ & 0.2 & 5.6 & 4.29 & 0.16 & $2.8 \mathrm{E}+36$ & $\mathrm{I}-\mathrm{B} 2 \mathrm{n}$ \\
\hline 9 & 40.662 & 1914.11 & $2.7 \pm 1.2$ & $3.70_{2.5}^{3.3}$ & 0 & 4.6 & 1.24 & 0.02 & $1.9 \mathrm{E}+37$ & II-C2n \\
\hline 18 & 41.076 & 1929.83 & $4.2 \pm 1.0$ & $3.92_{2.1}^{3.5}$ & 0.2 & 4.8 & 1.31 & 0.2 & $4.8 \mathrm{E}+37$ & $\mathrm{I}-\mathrm{Gn}$ \\
\hline 365 & 40.694 & 1909.93 & $4.1 \pm 0.8$ & $5.88_{2.6}^{4.5}$ & 0.18 & 4.0 & 3.22 & 0.14 & $5.1 \mathrm{E}+37$ & II-B \\
\hline 6 & 39.250 & 1933.95 & $4.4 \pm 0.8$ & $8.21_{7.1}^{77.1}$ & 0.14 & 4.0 & 2.68 & 0.12 & $9.7 \mathrm{E}+37$ & $\mathrm{I}-\mathrm{A} 1 \mathrm{n}$ \\
\hline 395 & 39.186 & 1930.32 & $2.1 \pm 0.7$ & $35.01_{2.9}^{3.1}$ & 0.12 & 4.6 & 7.03 & 0.14 & $9.0 \mathrm{E}+36$ & \\
\hline 1 & 39.442 & 1934.94 & $4.2 \pm 1.6$ & $94.00_{40.0}^{40.0}$ & 0.14 & 4.8 & 97.81 & 0.1 & $8.4 \mathrm{E}+37^{\mathrm{d}}$ & I-As \\
\hline
\end{tabular}

Notes.

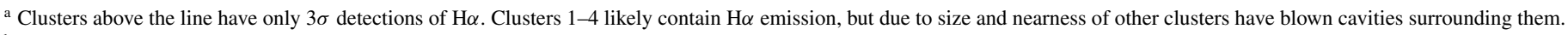

${ }^{b}$ Other names come from MacKenty et al. (2000).

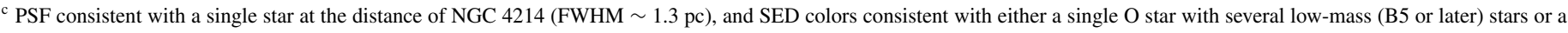
few early-B type stars.

${ }^{d}$ Luminosity derived from Table 3 in MacKenty et al. (2000). 
from both SLUG and SB99 for all 52 clusters; Figure 1 shows their placement in the galaxy. We have also included the cluster naming nomenclature used in MacKenty et al. (2000) where applicable for ease of comparison.

\section{DISCUSSION}

If the IMF is populated purely stochastically, then we can expect that $10010^{3} M_{\odot}$ clusters would contain the same numbers and masses of stars as one $10^{5} M_{\odot}$ cluster, and that both would represent a fully sampled IMF (Elmegreen 2001, 2006). On the other hand, if the most massive star in a cluster is limited by the mass of the parent cluster we might not expect any high-mass stars to be present in $1000 M_{\odot}$ clusters. For example, in the variable upper mass limit formulation of Weidner et al. (2010), they propose an $M_{\max *}-M_{\mathrm{cl}}$ relation in which no stars more massive than $35 M_{\odot}$ would be present in a $10^{3} M_{\odot}$ cluster. The summation of the total ionizing flux from these small clusters divided by the total cluster mass should then be much lower than the ionizing flux from the single large cluster divided by its mass, and as cluster mass decreases there is a deviation from the ratio of ionizing photons to mass expected by a universal IMF (Figure 5, dash-dotted line). In a universal IMF scenario, then, the summation of the total ionizing flux from the small clusters divided by the mass will be consistent with that of a single large cluster. Even though most low-mass $\left(\leqslant 500 M_{\odot}\right)$ clusters will produce low $\mathrm{H} \alpha$ luminosities in the universal scenario, there will be some that do produce a large ionizing continuum from the odd star well over $20 M_{\odot}$. Villaverde et al. (2010) estimate that only $20 \%$ of $100 M_{\odot}$ clusters will have stars large enough to create an $\mathrm{H}$ II region, therefore given a large enough sample the effects will average out. By summing the $L_{\mathrm{H} \alpha}$ and masses of all of the small clusters into one data point, not only are the observational uncertainties reduced but the stochastic effects are minimized.

We have used three mass bins (see Figure 5), each with a mean mass of $7.5 \times 10^{2} M_{\odot}, 2.2 \times 10^{3} M_{\odot}$, and $4 \times 10^{4} M_{\odot}$. The error bars in each bin are obtained by adding in quadrature the individual mass and luminosity errors of each cluster fit. The largest mass bin has only five members in total, including the super star cluster at the center of the galaxy (Cluster 1), for which the $\mathrm{H} \alpha$ luminosity is highly uncertain and we are using a value obtained in MacKenty et al. (2000). These extremely large clusters with negligible $\mathrm{H} \alpha$ emission are included to illustrate the possibility that we may have feedback occurring in our larger clusters which is dispersing the gas more efficiently. Indeed we note that the most massive star clusters in NGC 4214 are surrounded by ionized gas shells (see below). We have also included for illustration those clusters that have masses greater than $500 M_{\odot}$, but only upper limit measurements for $L_{\mathrm{H} \alpha}(1.6 \times$ $10^{35} \mathrm{erg} \mathrm{s}^{-1}$, open squares). Finally, we have included the full sample of objects with masses greater than $500 M_{\odot}$ with those objects which may be single stars or low-metallically clusters removed. In the lower mass bins, the error is dominated by the range of masses. Also plotted in Figure 5 is the expected average $L_{\mathrm{H} \alpha} / M_{\mathrm{cl}}$ from a $1 / 5 Z_{\odot} \mathrm{SB} 99$ metallicity model that is fully populated up to $120 M_{\odot}$ between $2-5 \mathrm{Myr}$ (top dashed line) and 2-8 Myr (bottom). The expected range for an $M_{*}-M_{\mathrm{cl}}$ model where the most massive star in the cluster is a function of cluster mass (Weidner et al. 2010) is shown in the dash-dotted line also averaged between 2-5 Myr (top) and 2-8 Myr (bottom). If the metallicity of NGC 4214 is $1 / 4 Z_{\odot}$ we do expect the data to fall somewhat below the stellar synthesis models, since lower metallicities create higher $\mathrm{H} \alpha$ luminosities.
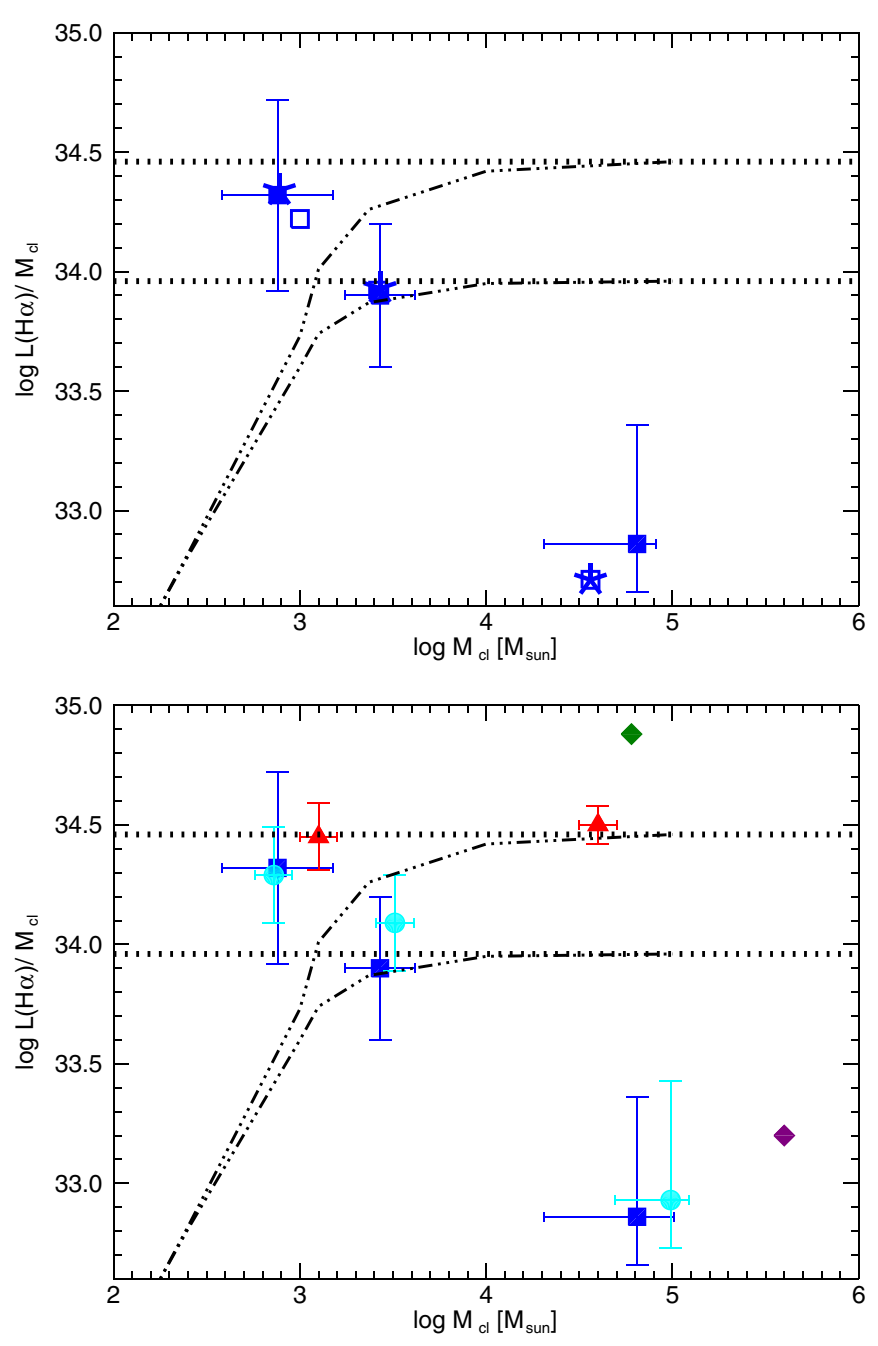

Figure 5. Top: location of $L_{\mathrm{H} \alpha} / M_{\mathrm{cl}}$ for mass bins in NGC 4214. Filled blue squares only include clusters with $\mathrm{H} \alpha$ emission (all of which are less than $6 \mathrm{Myr}$ ), while open blue squares include all clusters, and blue stars include all clusters without those objects which could possibly be single stars or lowmultiplicity clusters. The dotted line is the expected $L_{\mathrm{H} \alpha} / M_{\mathrm{cl}}$ for a universal IMF while the dash-dotted line is for a variable upper mass limit (Kroupa \& Weidner 2003; Weidner et al. 2010), where the most massive star in a cluster is determined by cluster mass. The top lines show averaged models between 2 and $5 \mathrm{Myr}$, while the bottom is between 2 and $8 \mathrm{Myr}$. Bottom: same as top, but also containing cluster measurements of NGC 4214 from SB99 (cyan circles), from M51 (Calzetti et al. 2010, red triangles), R136a (Pellegrini et al. 2012, green diamond), and NGC 330 (Pellegrini et al. 2012, purple diamond). The location of the largest mass blue and cyan symbols is highly impacted by the uncertainty of the $\mathrm{H} \alpha$ luminosity of Cluster 1.

(A color version of this figure is available in the online journal.)

When including upper limits in $\mathrm{H} \alpha$ the clusters in the highest mass bin have a value of $L_{\mathrm{H} \alpha} / M_{\mathrm{cl}}$ considerably lower than that of lower mass clusters (Figure 5). We have investigated whether the clusters in the highest mass bin may be experiencing feedback effects which have caused expulsion of the gas from the H II region. Pellegrini et al. (2012) have found that the amount of ionizing photons lost from a cluster can be dependent on the Hi density surrounding the cluster. With this in mind, we have used the H I maps of NGC 4214 published in Walter et al. (2001) to locate the clusters in the largest mass bin without $\mathrm{H} \alpha$ emission. We find that they are located in regions that have $\mathrm{H}$ I densities roughly $2 / 3$ that of the maximum density, which by itself would not indicate that the clusters should experience significant ionizing photon loss. When observing the clusters 


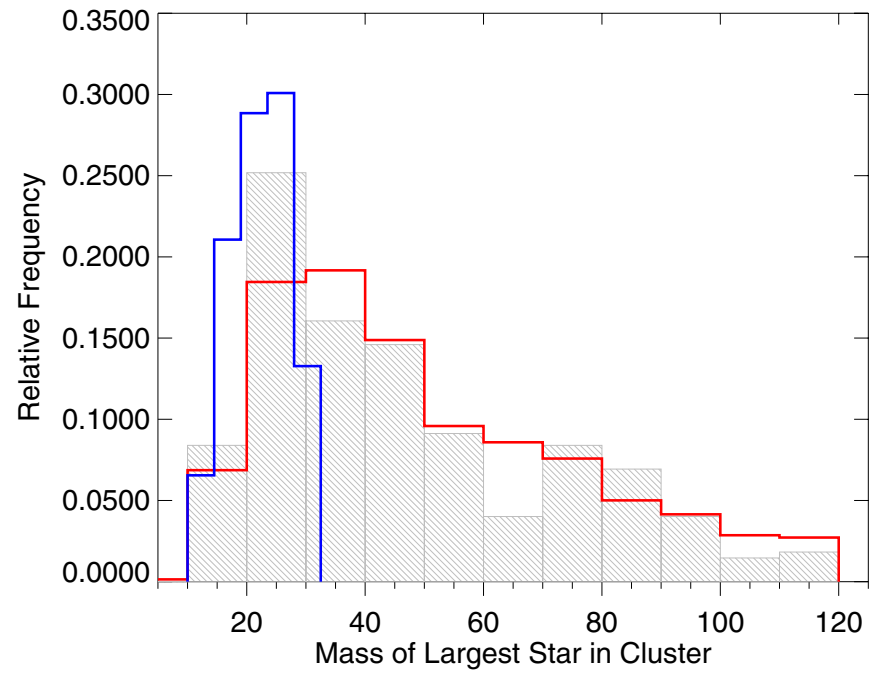

Figure 6. Histogram of the most massive star in each model considered the best fit along with fits within $\Delta \chi^{2}$ of $10 \%$ of the lowest value from SLUG plotted against a fully sampled IMF (red) and a model truncated at $30 M_{\odot}$ (blue). Masses range from 10 to $119 M_{\odot}$, and while $50 \%$ of the most massive stars are $<40 M_{\odot}$, there is still the existence of stars between 40 and $120 M_{\odot}$ in clusters with masses of $10^{3} M_{\odot}$ which would be contrary to a variable upper mass limit (Kroupa \& Weidner 2003; Weidner et al. 2010) theory.

(A color version of this figure is available in the online journal.)

in the $\mathrm{H} \alpha$ image though, it is quite clear that some mechanism has blown much of the gas away from the clusters and they are surrounded by wind-blown bubbles. This is not completely unexpected, as these clusters with extremely low $L_{\mathrm{H} \alpha}$ values are located in the NGC 4214-I region, in which Maíz Apellániz et al. (1998) found there was a significant decoupling of the stellar clusters with the ionized gas.

In Figure 4, we present histograms of the ages of the stellar clusters with detected $\mathrm{H} \alpha$ (blue) and non-detected $\mathrm{H} \alpha$ (red) in our sample, including one containing all clusters younger than $8 \mathrm{Myr}$ with masses $<10^{4} M_{\odot}$ (left), and all clusters with masses between 500 and $10,000 M_{\odot}$ (right). What we have found is that all clusters with ages that lie between 6 and 8 Myr have nondetections in $\mathrm{H} \alpha$. All clusters younger than 4.5 Myr are detected in $\mathrm{H} \alpha$, and $2 / 3$ of clusters between 4.5 and $6 \mathrm{Myr}$ are detected in $\mathrm{H} \alpha$. We must note that the uncertainties in each bin of Figure 4 are larger than the bin size, yet even when accounting for the uncertainties in the best-fit age, the ages of clusters showing $\mathrm{H} \alpha$ remain below $6 \mathrm{Myr}$. The exception here are those few clusters with masses $>10^{4} M_{\odot}$. These have not been included in Figure 4 because even at ages between 2 and $3 \mathrm{Myr}$, they have very little measured $\mathrm{H} \alpha$ flux, likely due to stronger feedback effects from the clusters themselves, or the more crowded environment in which they are located which makes it difficult to determine which ionizing photons come from which cluster. Relaño et al. (2012) have also determined that the leakage of ionizing photons is expected for those $\mathrm{H}$ II regions with ages greater than $4 \mathrm{Myr}$. While some of the detected clusters do venture into ages greater than $5 \mathrm{Myr}$, we do need to be aware of the fact that especially in clusters that may contain only one or two extremely massive stars they may not live long enough to produce ionizing photons out to $8 \mathrm{Myr}$. For example, the lifetime of a $35 M_{\odot}$ star is roughly 5 Myr, while a $15 M_{\odot}$ star may live $100 \mathrm{Myr}$ and a $120 M_{\odot}$ star only 2 Myr. If there is only one massive star in these smaller clusters, the $L_{\mathrm{H} \alpha}$ may be more sensitive to the age.

For each model, we show a histogram (Figure 6) of the most massive star in the model which best describes the photometry in each of the 47 clusters located in the two lowest mass bins. Included in this plot are also the error bars associated with the ensemble of fits within $\Delta \chi^{2}$ of $10 \%$ of the lowest value, and model lines for a fully sampled IMF (red) and a model truncated at $30 M_{\odot}$ (blue). What we find is that $25 \%$ of these model clusters have a maximum mass between 20 and $30 M_{\odot}$, $50 \%$ have a mass $<40 M_{\odot}$, and the remaining $50 \%$ contain most massive stars greater than $40 M_{\odot}$. We must point out that because clusters less than $500 M_{\odot}$ were excluded from the study it is possible that we may be underestimating the most massive stars with masses $<20 M_{\odot}$. According to Kroupa et al. (2011), specifically Figures 2 and 5, the expected maximum stellar mass of a cluster between 500 and $3000 M_{\odot}$ is between 20 and $40 M_{\odot}$ if the Weidner et al. (2010) hypothesis holds. Some of our clusters have a maximum stellar mass at this cluster mass in Figure 6 , but the existence of stellar masses greater than $40 M_{\odot}$ for the same range of cluster masses indicates that there is no maximum mass for the stars in these clusters, other than the usual upper limit found in much more massive clusters. Our results in Figure 6 are consistent with the random sampling from Figure 2 of Kroupa et al. (2011), where the maximum stellar mass can range between 15 and $120 M_{\odot}$ for a 500-3000 $M_{\odot}$ cluster. We must point out that these models were populated specifically for $1 \times 10^{3} M_{\odot}$ clusters, and the clusters themselves range between 500 and $9000 M_{\odot}$, but that our treatment should give a reasonable representation of the actual spread of massive stars. As mentioned above, tests run on $500 M_{\odot}, 1000 M_{\odot}$, and $3000 M_{\odot}$ SLUG models resulted in mass and age discrepancies within the uncertainties of the $\chi^{2}<1$ results.

Larsen (2002) has suggested that lower SFR correlates with a lower average cluster mass; therefore, most galaxies with a low SFR should be deficient in high-mass clusters in a purely stochastic case. For example, 100 galaxies with an SFR of $0.1 M_{\odot} \mathrm{yr}^{-1}$ will have the same distribution of cluster masses as one galaxy with an SFR of $10 M_{\odot} \mathrm{yr}^{-1}$, yet most of the low SFR galaxies will be lacking massive clusters and a few will have an overabundance. Other studies have indicated that there is a systematic trend for small clusters to form only low-mass stars (Kroupa \& Weidner 2003; Weidner et al. 2010; PflammAltenburg et al. 2009, for example), effectively steepening the slope of the uIMF (Meurer et al. 2009). When interpolated across a galaxy, the integrated galactic IMF (Kroupa \& Weidner 2003; Weidner et al. 2010, IGIMF) would ultimately lead to low SFR galaxies such as dwarfs to be deficient in both large stellar clusters and massive stars. From what we have presented above, we do not find that this is the case in NGC 4214. The SLUG models suggest that roughly $50 \%$ of clusters with masses around $1 \times 10^{3} M_{\odot}$ have at least one star more massive than $40 M_{\odot}$ (Figure 6). We have also performed a Kolmogorov-Smirnov (K-S) test between the best-fit $L_{\mathrm{H} \alpha} / M_{\mathrm{cl}}$ data and both SLUG models sampled up to $30 M_{\odot}$ and $120 M_{\odot}$ (blue and red lines in Figure 7 , respectively). We find that there is about a $65 \%$ chance the data were drawn from the parent model of the fully populated Kroupa IMF up to $120 M_{\odot}$, but only about a $1 \%$ chance they come from the models truncated at $30 M_{\odot}$. By adding in error bars from the ensemble of fits within $\Delta \chi^{2}$ (right), these numbers become $75 \%$ and $1 \%$, respectively. When we compare the $L_{\mathrm{H} \alpha} / M_{\mathrm{cl}}$ values of the clusters which were age-dated using an SB99 model where the most massive star is only allowed to be $30 M_{\odot}$ against the fully populated and truncated SLUG models (see Figure 7, bottom) the K-S test only shows agreement of $7 \%$ and $4 \%$ respectively that the data come from those respective populations, even when uncertainties are included. 

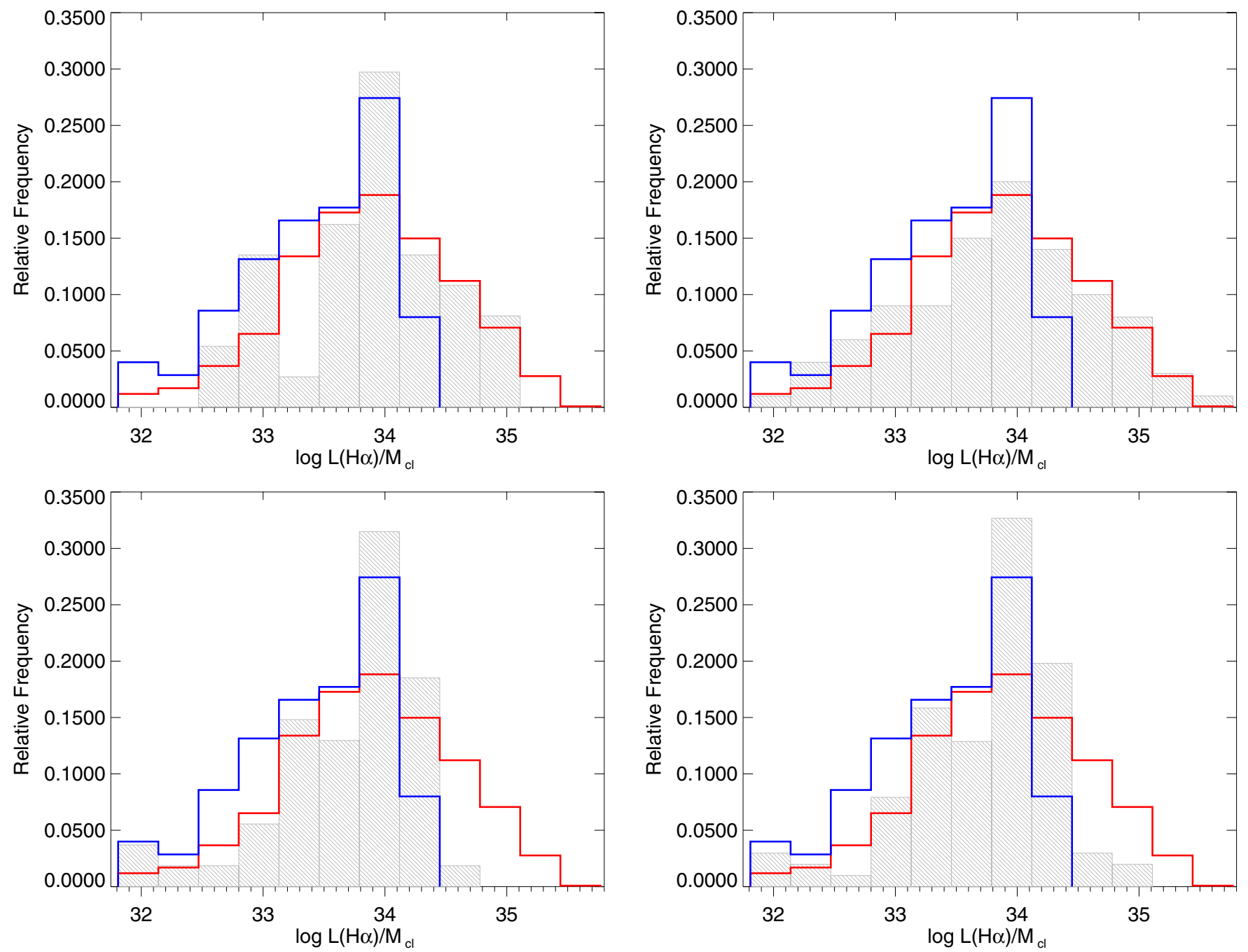

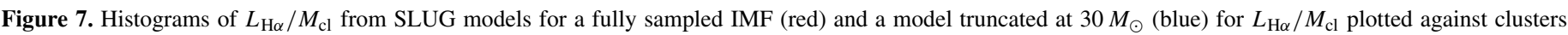

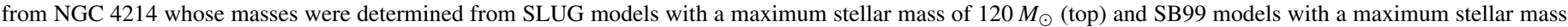
of $30 M_{\odot}$ (bottom). The left panels only include the single best-fit mass, while the right panels include error bars for all masses with a $\chi^{2}<1$.

(A color version of this figure is available in the online journal.)

Thus, even allowing our clusters to be modeled as drawn from a parent population that does not have stars more massive than $30 M_{\odot}$ (which affect the determination of the cluster masses) the $L_{\mathrm{H} \alpha} / M_{\mathrm{cl}}$ distribution does not change in such a way as to agree with a truncated IMF.

\section{CONCLUSIONS}

We have attempted to constrain the upper end of the IMF of the nearby irregular star-bursting dwarf galaxy NGC 4214 using the method of Calzetti et al. (2010), which uses the ratio of the luminosity of the ionizing photons normalized to the mass of the cluster as a proxy for probing the presence of massive stars. With a final sample of 52 young clusters with masses $>500 M_{\odot}$, we have determined that even at masses $\sim 10^{3} M_{\odot}$, there does not seem to be a deviation from the expected ionizing flux of a universal IMF up to $120 M_{\odot}$. Clusters with a mean mass down to $700 M_{\odot}$ have an $L_{\mathrm{H} \alpha} / M_{\mathrm{cl}}$ ratio that lies along that predicted by a universal IMF.

We have also determined that a truncated IMF (one in which the maximum $M_{*}$ is a function of $M_{\mathrm{cl}}$ ), which would result in the maximum stellar mass in a cluster of $10^{3} M_{\odot}$ being no greater than $35 M_{\odot}$ (Weidner et al. 2010), does not sufficiently explain the young clusters seen in NGC 4214. Models used to age-date the clusters indicate that up to $50 \%$ of the clusters contain a massive star greater than $40 M_{\odot}$, while $\mathrm{K}-\mathrm{S}$ tests indicate that there is only a $1 \%$ chance that the $L_{\mathrm{H} \alpha} / M_{\mathrm{cl}}$ values from clusters in NGC 4214 come from a parent model with the maximum mass truncated at $30 M_{\odot}$ and a $75 \%$ chance that they arise from a fully populated IMF up to $120 M_{\odot}$. As with Fumagalli et al. (2011), who investigated the integrated properties of individual galaxies, we find that the summation of individual young clusters in NGC 4214 is more consistent with a universal IMF without a truncation of massive stars. Our test shows not only that the $M_{*}-M_{\mathrm{cl}}$ relation of Weidner et al. (2010) is in disagreement with the observations, but also that the data are consistent with a stochastically sampled IMF with our upper mass limit consistent with a standard Kroupa (2001) IMF.

We thank the referee for valuable comments on the paper. J.A. and D.C. acknowledge partial support for this study from the grant associated with program GO-11360 (PI: R. W. O'Connell), which was provided by NASA through the Space Telescope Science Institute, which is operated by the Association of Universities for Research in Astronomy, Inc., under NASA contract NAS 5-26555. M.R.K. acknowledges support from an Alfred P. Sloan Fellowship, from the NSF 
through grant CAREER-0955300, and from NASA through a Chandra Space Telescope Grant and through Astrophysics Theory and Fundamental Physics Grant NNX09AK31G. The work of R.L.dS. is supported by a National Science Foundation Graduate Research Fellowship.

\section{APPENDIX}

\section{FILTER CONVOLUTION}

SLUG models, as of the submission of this paper, are not delivered in HST filter passbands. Luminosities were delivered in Johnson-Cousins $U B V I$ and Galex NUV, and, because of this, transformation coefficients were derived to approximate WFC3 filters. To derive these coefficients, each 1 Myr time step of the SB99 model was convolved with the appropriate WFC3 filter passband (discussed below) to get the fluxes in each filter at each age. The SLUG models were then divided into $1 \mathrm{Myr}$ age bins (for example, the $3 \mathrm{Myr}$ age bin consisted of all models between the ages of 2.5 and $3.4 \mathrm{Myr}$ ), and the flux in each filter was averaged into a mean flux. Therefore for $1 \mathrm{Myr}$ age bins, there exists an average luminosity for each filter to directly compare with SB99 models. The ratio between the SB99 and SLUG models was then taken, resulting in coefficients to convert SLUG filters to WFC3 filters. These values were then applied back onto each of the individual $\sim 40,000$ SLUG models, and the effective wavelengths were adjusted to reflect those of WFC3 filters.

For the SB99 models, the filter convolution process was more straightforward. From the output SED each 1 Myr age bin was extracted and then using the IRAF function sinterp they were all interpolated into the same wavelength range of 500-10000. . Each WFC3/UVIS filter transmission curve was also extracted into the identical wavelength range, and then integrated over those wavelengths to determine the total transmission for each filter at the effective wavelength $\left(\int T_{\lambda} d \lambda\right)$. The SB99 SEDs were then multiplied by the transmission curves and a numerical integrated value was obtained $\left(\int F_{\lambda} T_{\lambda} d \lambda\right)$. Next, $\left\langle F_{\lambda}\right\rangle=\int F_{\lambda} T_{\lambda} d \lambda / \int T_{\lambda} d \lambda$ is then calculated for each age bin and each filter. This transforms the continuous SB99 SED into five distinct photometry points which can be easily compared with the photometry of the NGC 4214 clusters (Figure 3).

Once the models have been convolved with the filters, they are then corrected for possible host galaxy extinction. Due to the low metallically of this galaxy, we have chosen to use an SMC extinction curve from Fitzpatrick (1999) which is more in line with the metallically of NGC 4214 . We have limited our reddening to lie between $0.0 \leqslant E(B-V) \leqslant 0.40$ as previous studies on this galaxy seem to indicate low values of extinction (Úbeda et al. 2007b). The population synthesis SEDs are first convolved with the extinction curve at selected values of the color excess $E(B-V)$, spanning the full range of $E(B-V)$ used for our analysis $(0.0-0.4)$, in order to determine the effective wavelengths $\lambda_{\text {ext,eff }}$ to be used when applying extinction to broadband filter photometry. In general, $\lambda_{\text {ext,eff }}$ is different from the effective wavelength of a filter, owing to the non-symmetric transmission curves of most filters. We should note that $\lambda_{\text {ext,eff }}$ depends also on the range of $E(B-V)$ considered and should be recalculated when using color excesses outside our range. We elect to apply extinction corrections after filter convolution because tests comparing the results of this procedure against the other procedure of applying the extinction before filter convolution give differences of only $5 \%$. Our choice, however, provides the flexibility to change $E(B-V)$ at will. Each filter is then multiplied by $e^{0.92 \times E(B-V) \times \mathrm{SMC}}$, where $E(B-V)$ is in increments of 0.02 , and the appropriate SMC extinction value is determined for the wavelength of each filter.

\section{REFERENCES}

Anderson, J., Piotto, G., King, I. R., Bedin, L. R., \& Guhathakurta, P. 2009, ApJL, 697, L58

Ascenso, J., Alves, J., \& Lago, M. T. V. T. 2009, A\&A, 495, 147

Bastian, N., Covey, K. R., \& Meyer, M. R. 2010, ARA\&A, 48, 339

Boselli, A., Boissier, S., Cortese, L., et al. 2009, ApJ, 706, 1527

Calzetti, D., Armus, L., Bohlin, R. C., et al. 2000, ApJ, 533, 682

Calzetti, D., Chandar, R., Lee, J. C., et al. 2010, ApJL, 719, L158

Cappellari, M., McDermid, R. M., Alatalo, K., et al. 2012, Natur, 484, 485

Cerviño, M., \& Luridiana, V. 2004, A\&A, 413, 145

Cerviño, M., \& Luridiana, V. 2006, A\&A, 451, 475

Chandar, R., Whitmore, B. C., Calzetti, D., et al. 2011, ApJ, 727, 88

Chandar, R., Whitmore, B. C., Kim, H., et al. 2010, ApJ, 719, 966

Corbelli, E., Verley, S., Elmegreen, B. G., \& Giovanardi, C. 2009, A\&A, 495, 479

da Silva, R. L., Fumagalli, M., \& Krumholz, M. 2012, ApJ, 745, 145

Dopita, M. A., Calzetti, D., Maíz Apellániz, J., et al. 2010, Ap\&SS, 330, 123

Eldridge, J. J. 2012, MNRAS, 422, 794

Elmegreen, B. G. 2001, in ASP Conf. Ser. 243, From Darkness to Light: Origin and Evolution of Young Stellar Clusters, ed. T. Montmerle \& P. André (San Francisco, CA: ASP), 255

Elmegreen, B. G. 2006, ApJ, 648, 572

Fardal, M. A., Katz, N., Weinberg, D. H., \& Davé, R. 2007, MNRAS, 379,985

Fitzpatrick, E. L. 1999, PASP, 111, 63

Fumagalli, M., da Silva, R. L., \& Krumholz, M. R. 2011, ApJL, 741, L26

Gunawardhana, M. L. P., Hopkins, A. M., Sharp, R. G., et al. 2011, MNRAS, 415, 1647

Hoversten, E. A., \& Glazebrook, K. 2008, ApJ, 675, 163

Kennicutt, R. C., Jr., Lee, J. C., Funes, José G., et al. 2008, ApJS, 178, 247

Kobulnicky, H. A., \& Skillman, E. D. 1996, ApJ, 471, 211

Kroupa, P. 2001, MNRAS, 322, 231

Kroupa, P., \& Weidner, C. 2003, ApJ, 598, 1076

Kroupa, P., Weidner, C., Pflamm-Altenburg, J., et al. 2011, arXiv:1112.3340

Lada, C. J., \& Lada, E. A. 2003, ARA\&A, 41, 57

Larsen, S. S. 2002, AJ, 124, 1393

Lee, J. C., Gil de Paz, A., Kennicutt, R. C., Jr., et al. 2011, ApJS, 192, 6

Lee, J. C., Gil de Paz, A., Tremonti, C., et al. 2009, ApJ, 706, 599

Leitherer, C., Schaerer, D., Goldader, J. D., et al. 1999, ApJS, 123, 3

Leitherer, C., Vacca, W. D., Conti, P. S., et al. 1996, ApJ, 465, 717

MacKenty, J. W., Maíz-Apellániz, J., Pickens, C. E., Norman, C. A., \& Walborn, N. R. 2000, AJ, 120, 3007

Maíz Apellániz, J. 2008, ApJ, 677, 1278

Maíz Apellániz, J., Mas-Hesse, J. M., Munoz-Tunon, C., Vilchez, J. M., \& Castaneda, H. O. 1998, A\&A, 329, 409

Massey, P. 2003, ARA\&A, 41, 15

Meurer, G. R., Wong, O. I., Kim, J. H., et al. 2009, ApJ, 695, 765

Oey, M. S. 2011, ApJL, 739, L46

Pasquali, A., de Grijs, R., \& Gallagher, J. S. 2003, MNRAS, 345, 161

Pellegrini, E. W., Oey, M. S., Winkler, P. F., et al. 2012, ApJ, 755, 40

Pflamm-Altenburg, J., Weidner, C., \& Kroupa, P. 2009, MNRAS, 395, 394

Popescu, B., \& Hanson, M. M. 2010, ApJ, 724, 296

Reines, A. E., Nidever, D. L., Whelan, D. G., \& Johnson, K. E. 2010, ApJ, 708,26

Relaño, M., Kennicutt, R. C., Jr., Eldridge, J. J., Lee, J. C., \& Verley, S. 2012, MNRAS, 423, 2933

Sabbi, E., Sirianni, M., Nota, A., et al. 2008, AJ, 135, 173

Sirianni, M., Nota, A., Leitherer, C., De Marchi, G., \& Clampin, M. 2000, ApJ, 533, 203

Úbeda, L., Maíz-Apellániz, J., \& MacKenty, J. W. 2007a, AJ, 133, 917

Úbeda, L., Maíz-Apellániz, J., \& MacKenty, J. W. 2007b, AJ, 133, 932

van Dokkum, P. G., \& Conroy, C. 2011, ApJL, 735, L13

Villaverde, M., Cerviño, M., \& Luridiana, V. 2010, A\&A, 522, A49

Walter, F., Taylor, C. L., Hüttemeister, S., Scoville, N., \& McIntyre, V. 2001, AJ, 121,727

Weidner, C., Kroupa, P., \& Bonnell, I. A. D. 2010, MNRAS, 401, 275

Weisz, D. R., Johnson, B. D., Johnson, L. C., et al. 2012, ApJ, 744, 44

Whitmore, B. C., Chandar, R., Kim, H., et al. 2011, ApJ, 729, 78

Wilkins, S. M., Hopkins, A. M., Trentham, N., \& Tojeiro, R. 2008, MNRAS, 391, 363

Williams, B. F., Dalcanton, J. J., Gilbert, K. M., et al. 2011, ApJ, 735, 22 